\title{
Nonlinear dynamics of multiple neoclassical tearing modes in tokamaks
}

\author{
D. Chandra, ${ }^{1}$ O. Agullo, ${ }^{2}$ S. Benkadda,${ }^{2}$ X. Garbet, ${ }^{3}$ and A. Sen ${ }^{1}$ \\ ${ }^{1}$ Institute for Plasma Research, Bhat, Gandhinagar 382428, India \\ ${ }^{2}$ France-Japan Magnetic Fusion Lab, LIA 336, CNRS/Lab PIIM, UMR 6633, CNRS-Universite de Provence, \\ 13397 Marseille, France
}

Association Euratom-CEA, DRFC, CEA Cadarache, 13108 St-Paul-Lez-Durance, France

Numerical simulations investigating the interaction of co-existent $2 / 1$ and $3 / 1$ neoclassical tearing modes are presented. The results obtained from an initial value 3D toroidal code that solves a set of generalized reduced MHD equations exhibit a host of complex phenomena arising from the coupling of the two modes. These include a modification of the island saturation widths of the two modes, a significant modification in the perpendicular flow patterns in the vicinity of the islands,

and the excitation of geodesic acoustic mode like oscillations that lead to concomitant oscillations in the kinetic and magnetic energies of the islands. These oscillations only occur in the presence of the neoclassical stress tensor contribution and are absent for two coupled classical tearing modes.

\section{INTRODUCTION}

Neoclassical tearing modes (NTMs) pose a serious challenge for the optimal operation of advanced tokamaks like ITER $^{1,2}$ that would like to operate at high values of $\beta$ in order to maximize their efficiency. These modes are excited by the loss of the bootstrap current inside a seed magnetic island causing the island to grow. If the growth is not controlled it can lead to severe degradation of confinement and in some cases to disruptions. NTMs are believed to set the upper limit on attainable normalized plasma $\beta_{N}{ }^{3,4}$ in long pulse tokamaks and have therefore been the subject of much experimental and theoretical investigations in the recent past. ${ }^{5-11}$ While there has been considerable progress in the experimental characterization and control of this instability with concurrent development of a useful theoretical framework in the form of a model island evolution equation (the modified Rutherford equation) there are still many basic physics issues associated with the mode that remain poorly understood or relatively less explored. The trigger and the mechanism for the creation of the seed island, nonlinear mode coupling between NTMs of different helicities, interaction of the magnetic island with energetic particles, evolution of the island in a turbulent media $^{12-14}$ are among some of the basic areas that need further attention. Mode coupling is an important issue that can play a significant role at various stages of the evolution of NTMs starting from the onset to its saturation. The triggering of a seed island at the $q=2$ surface due to a sawtooth crash at the $q=1$ surface or due to fishbones is an example of a mode coupling process operating at the onset stage. ${ }^{5,6}$ There is also experimental evidence of mode coupling effects influencing the evolution of multiple NTMs often leading to the predominance of a single helicity over others. ${ }^{6,8-10}$ There have been some past model studies addressing the issue of the interaction between multiple helicity NTMs. In Ref. 7, it was suggested that the interaction of magnetic islands produces a stochastic region around the separatrices of the islands. This interaction causes the island pressure profile to be broadened, reducing the island bootstrap current and drive for the magnetic island. The creation of a stochastic region requires the islands to be close to each other creating an overlap between them. In a similar study in Ref. 8, it was shown that once two magnetic islands of different helicities got close to each other the more unstable magnetic island survived and suppressed the less unstable one. The mechanism responsible for this suppression was attributed to the decreased fundamental harmonic pressure perturbations of the NTM in the presence of magnetic perturbations of different helicities. The role of a stochastic layer was also emphasized in Ref. 9 where for MHD simulations carried out for ITER like plasmas it was found that the modes do not couple until significant stochastization between two tearing island chains occurs. After stochastization a quick amplitude drop of one or several modes was observed. In the present work, we revisit this problem and study the evolution of two unstable NTMs with well separated resonant surfaces (of helicities $2 / 1$ and 3/1) through numerical simulations carried out on a fully toroidal code that solves a set of generalized MHD equations. ${ }^{11,15}$ In contrast to earlier studies, we find that there can be significant coupling between the two modes without the creation of a stochastic layer and resulting in quite complex nonlinear behavior. The coupling is found to occur through modifications of the velocity flow patterns around the magnetic islands which induce geodesic acoustic mode (GAM)-like oscillations of the mode energies in the presence of strong $(0 / 0)$ and (1/0) zonal magnetic fields. Such a phenomenon is observed only for NTMs and not for two classical tearing modes evolving simultaneously indicating that the mechanism is related to pressure perturbations modifying the neoclassical drive. These oscillations also disappear in the absence of $V_{\|}$dynamics. The coupling reduces the saturation states of both the modes without fully suppressing the weakly unstable mode. Such an alternate coupling mechanism might explain the existence of multiple NTMs in some recent experiments on $\mathrm{JET}^{16}$ where simultaneous existence of (2/1) and (3/1) modes have been observed. 


\section{MODEL EQUATIONS}

As mentioned above, our numerical simulations are based on the solutions of a set of generalized reduced MHD equations that were first derived in Ref. 15. The equations, which are valid for any aspect ratio, were derived using $k_{\|} / k_{\perp}$ (the ratio of parallel to perpendicular wave numbers) as a small expansion parameter $\delta$ and by employing a multiple scale analysis that respects equilibrium constraints and also permits elimination of fast time scales associated with perpendicular wave motion. The model equations thus evolve scalar potential quantities on a time scale associated with the parallel wave vector (shear-Alfvén wave time scale), which is the time scale of interest for resistive MHD instabilities like tearing modes. In the limit of $\beta \sim \delta^{1 / 2}(\delta \ll 1$ ) (where $\beta$ is the ratio of plasma pressure to magnetic pressure), the evolution equations can be written as follows:

$$
\begin{gathered}
\frac{\partial \Psi}{\partial t}-\left(\vec{b}_{0}+\vec{b}_{1}\right) \cdot \nabla \phi_{1}-\vec{b}_{1} \cdot \nabla \phi_{0}=\eta J_{\| 1}-\frac{1}{n e} \vec{b}_{0} \cdot \nabla \cdot \vec{\Pi}_{e} \\
\nabla \cdot\left(\frac{\rho}{B_{0}} \frac{d}{d t} \frac{\nabla \phi_{1}}{B_{0}}\right)+\left(\vec{V}_{1} \cdot \nabla\right)\left(\nabla \cdot\left(\frac{\rho}{B_{0}} \frac{\nabla \phi_{0}}{B_{0}}\right)\right) \\
=\left(\vec{B}_{0} \cdot \nabla\right) \frac{J_{\| 1}}{B_{0}}+\left(\vec{B}_{1} \cdot \nabla\right) \frac{J_{T \|}}{B_{0}} \\
\frac{d p_{1}}{d t}+\left(\vec{V}_{1} \cdot \nabla\right) p_{0}+\Gamma p_{0} \nabla \cdot \vec{V}_{\| 1}+\Gamma p_{0} \nabla \cdot \vec{V}_{\perp 1} \\
+\Gamma p_{1} \nabla \cdot \vec{V}_{1}=-(\Gamma-1) \nabla \cdot \vec{q} \\
\frac{\partial V_{\| 1}}{\partial t}+\left(\vec{V}_{\perp 1} \cdot \nabla\right) V_{\| 1}+\left(\vec{V}_{\| 1} \cdot \nabla\right) V_{\| 1} \\
=-\vec{b}_{0} \cdot \nabla p_{1}-\vec{b}_{1} \cdot \nabla p_{0}-\vec{b}_{1} \cdot \nabla p_{1} \\
\quad+\chi_{\perp} \nabla p_{1}-\left(\chi_{\|}-\chi_{\perp}\right)\left[\vec{b}_{1} \cdot \nabla\left(\vec{b}_{0} \cdot \nabla p_{0}\right)\right. \\
\quad+\vec{b}_{0} \cdot \nabla\left(\vec{b}_{0} \cdot \nabla p_{1}+\vec{b}_{1} \cdot \nabla p_{0}\right) \\
+\vec{b}_{0} \cdot \nabla\left(\vec{b}_{1} \cdot \nabla p_{1}\right)+\vec{b}_{1} \cdot \nabla\left(\vec{b}_{1} \cdot \nabla p_{0}\right) \\
\left.+\vec{b}_{1} \cdot \nabla\left(\vec{b}_{0} \cdot \nabla p_{1}\right)\right]
\end{gathered}
$$

where subscript 0 denotes equilibrium quantities and subscript 1 denotes perturbed quantities. Further,

$$
\begin{aligned}
& \frac{d}{d t}=\frac{\partial}{\partial t}+\vec{V} \cdot \nabla ; \quad \vec{V}=\frac{\vec{B}_{0} \times \nabla \phi_{1}}{B_{0}^{2}}+V_{\| 1} \vec{b}_{T} ; \\
& \vec{b}_{0}=\frac{\overrightarrow{B_{0}}}{B}, \quad \vec{b}_{1}=\frac{\overrightarrow{B_{1}}}{B}, \quad \vec{b}_{T}=\vec{b}_{0}+\vec{b}_{1}, \\
& \nabla \cdot \overrightarrow{\vec{\Pi}}_{e}=\rho_{e} \mu_{e}\left\langle B^{2}\right\rangle \frac{\vec{v}_{e} \cdot \nabla \theta}{(\vec{B} \cdot \nabla \theta)^{2}} \nabla \theta ; \\
& \left.\vec{v}_{e}=-\frac{1}{n e} \quad J_{\| 1} \vec{b}_{0}+\frac{\vec{B}_{0} \times \vec{\nabla} p_{1}}{B_{0}^{2}}\right) ; \quad J_{\| T}=J_{\| 0}+J_{\| 1}, \\
& \vec{B}_{0} \cdot \vec{\nabla} \cdot \overrightarrow{\Pi_{e}}=-\frac{\rho_{e} \mu_{e}}{n e}\left\langle B^{2}\right\rangle J_{\theta} ; \quad J_{\theta}=\frac{\nabla^{2} \Psi B_{0}}{B_{0}^{2}}+\frac{I}{B_{0}^{2}} \frac{\partial p}{\partial \psi_{0}} ; \\
& J_{\| 0}=\frac{\vec{J}_{0} \cdot \overrightarrow{B_{0}}}{B} ; \quad J_{\| 1}=\frac{\vec{J}_{1} \cdot \overrightarrow{B_{0}}}{B} .
\end{aligned}
$$

Equation (1) is Ohm's law, with $\Psi$ representing the magnetic flux, $\phi$ is the electrostatic potential, $J$ is the current density, $\eta$ is the resistivity, $B$ is the magnetic field, and $\overrightarrow{\vec{\Pi}}_{e}$ is the electron stress tensor. The last term on the right hand side of Ohm's law is the contribution from the neoclassical electron viscous damping term and is the driving term for the neoclassical tearing mode. Equation (2) is the vorticity equation with $\vec{V}$ the flow velocity. Equation (3) is the pressure evolution equation where heat flow terms have been retained with $\vec{q}$ representing the heat flux, $\Gamma$ representing the ratio of specific heats and $\chi_{\perp}, \chi_{\|}$representing the heat transport coefficients in the perpendicular and parallel directions, respectively. In general the transport coefficients can depend on various plasma parameters such as temperature, density, turbulence levels as well as kinetic effects. For the evolution of NTMs, we have assumed that in the vicinity of the island structure the effect of topological changes associated with the magnetic island are likely to be more important than the spatial variation of the coefficients. ${ }^{17} \mathrm{We}$ have, therefore, modeled $\chi_{\|}$and $\chi_{\perp}$ to be constant across the plasma radius. The critical parameter is the ratio $\chi_{\|} / \chi_{\perp}$ which has to be large enough to ensure rapid plasma pressure equilibration on the flux surface. ${ }^{11}$ For all our runs, we have ensured that this happens by keeping the ratio to be $\geq 10^{6}$. Finally, Equation (4) is the evolution equation for the parallel velocity component. Here, the mass density $\rho$ has been taken as a constant. The above equations have been programmed into an initial value code, called NEAR, which was benchmarked and used for classical and neoclassical tearing modes in previous studies. ${ }^{11}$ The various details of the code like normalization, algorithm etc., have been described in Refs. 11 and 15. Our emphasis in the present work is on understanding the nonlinear dynamics of multiple tearing modes in the presence of the neoclassical viscous stress tensor term in Ohm's law. The self consistent equilibrium for all our studies were generated by numerically solving the Grad Shafranov (GS) equation with the help of the TOQ code. ${ }^{18}$ This code solves the GS equation using an inverse equilibrium solver in which the real space coordinates are directly obtained as functions of the magnetic flux functions. The coordinate system used in the code is of the constant arc length variety as opposed to a straight field line representation. An iterative algorithm is employed to obtain a solution when given any two flux functions out of various options, such as specifying the pressure gradient profile and the q profile, or the pressure gradient profile and parallel current density profile etc. In our case we have specified a pressure profile of the form $p_{0}=p_{00}\left(1-x^{2}\right)^{2.49}$, where $x=r / a$ is the normalized minor radial coordinate, $p_{00}$ is the maximum value of the pressure, and $a$ is the minor radius. The parallel current density profile has been taken to be of the form $J_{\| 0}=J_{\| 00}\left(1-1.41 x^{2}\right.$ $\left.+0.439 x^{4}-0.65 x^{6}+1.62 x^{8}-1.68 x^{10}+0.685 x^{12}\right), \quad$ where $J_{\| 00}$ is the peak value of the parallel current density. The normalized radial profiles of the equilibrium pressure, the equilibrium parallel current density and the corresponding profile of the safety factor $\mathrm{q}$ are shown in Fig. 1.

\section{NUMERICAL SIMULATION RESULTS}

In this section, we describe the simulation results obtained using NEAR. We have taken $(m, n)=(2,1)$ and 

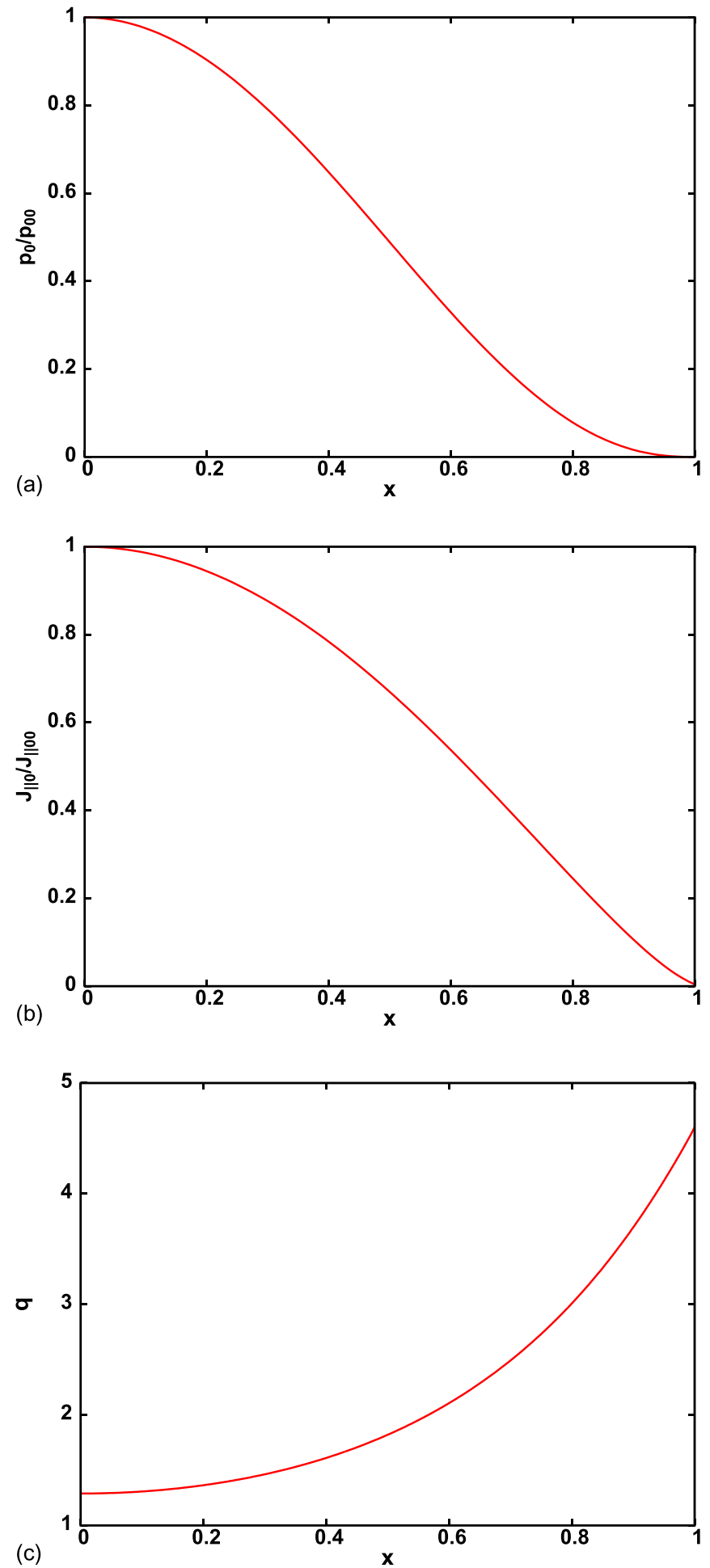

FIG. 1. Normalized equilibrium profiles for the plasma pressure (top panel), parallel current density (center panel), and the safety factor (bottom panel).

$(3,1)$ as perturbed modes, where $\mathrm{m}$ is the poloidal mode number and $\mathrm{n}$ is the toroidal mode number. A large number of equilibrium modes $(m, n)=(0,0),(1,0),(2,0),(3,0),(4,0)$, etc. have been evolved to take care of the nonlinear modification of the equilibrium. The choice of these modes is motivated by recent experimental observations of the simultaneous existence of $(2,1)$ and $(3,1)$ modes in the JET tokamak. ${ }^{16}$ Further, as will become clear later in the paper, the nonlinearly excited $(1,0)$ mode plays an important role and its excitation gets naturally enhanced due to coupling between modes with the same toroidal mode number. The magnetic Reynolds number $\mathrm{S}\left(=\tau_{R} / \tau_{A}\right)$ has been kept at $10^{5}$ in all runs. We have taken a typical equilibrium of circular shape with aspect ratio $\mathrm{R} / \mathrm{a} \sim 10$ with the equilibrium profiles mentioned in Sec. II and as shown in Fig. 1. This particular value of the aspect ratio was chosen for convenience since the code has been validated extensively for such a value in our earlier simulations. ${ }^{11,19}$ As shown in Ref. 15, the model equations we have used do not rely for their derivation on a large aspect ratio assumption, and hence a change in the aspect ratio to smaller values is not likely to have a significant impact on the results obtained.

At first, we have started with purely neoclassical tearing modes. We have taken an equilibrium profile (with toroidal $\left.\beta_{0}=0.009\right)$ that is classically stable $\left(\Delta^{\prime}<0\right)$ to the $(2,1)$ and $(3,1)$ modes. We have made the equilibrium classically stable or unstable by changing the plasma $\beta$ values as $\Delta^{\prime}$
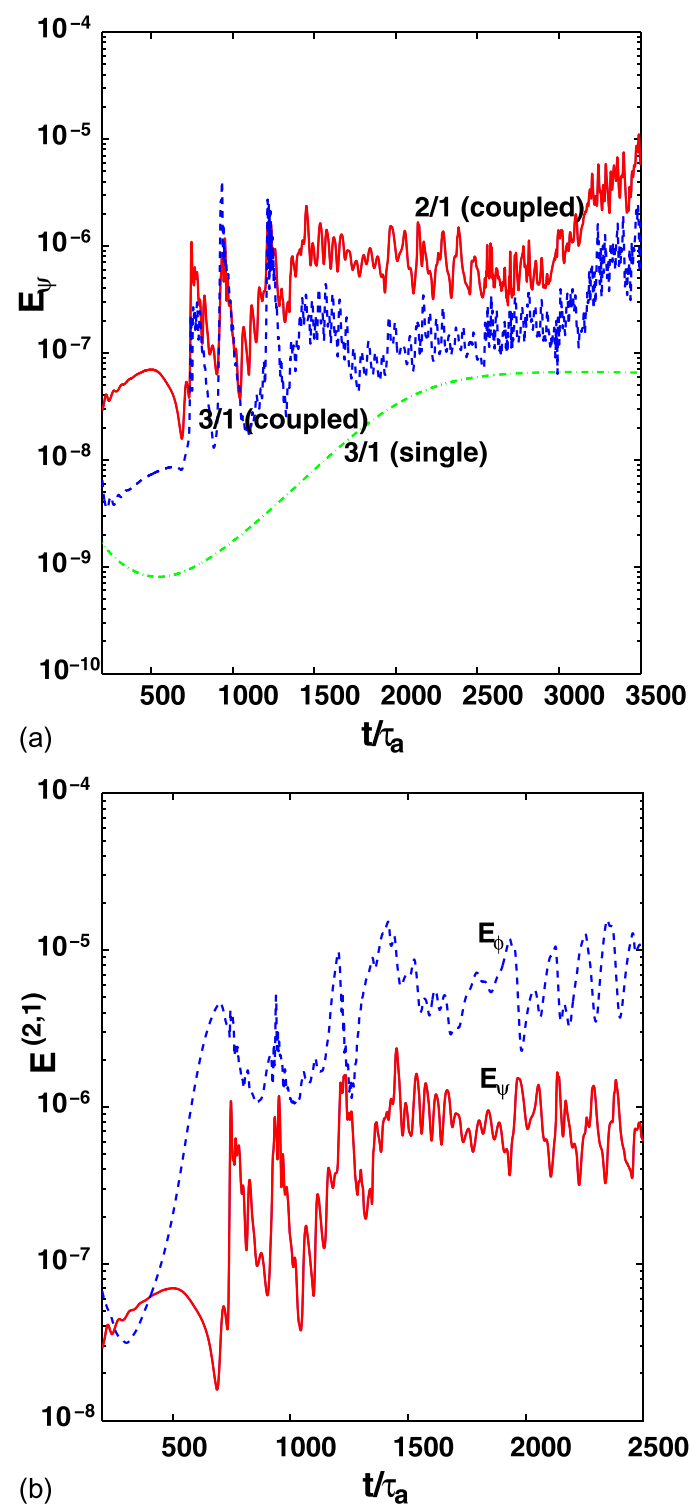

FIG. 2. Temporal evolution of magnetic energies for coupled and single NTMs (top panel) and comparison of magnetic and flow energies (bottom panel) for $\Delta^{\prime}<0$, finite $\mu_{e}$. 
decreases with the increase of the plasma $\beta$ value. So in the absence of any neoclassical driving term $\left(\mu_{e}=0\right)$ or at very low initial amplitudes the mode energies decrease as they are evolved in time. In the presence of a finite $\mu_{e}$ and for an initial amplitude beyond a threshold value the mode is unstable and starts to grow till it saturates nonlinearly. In Fig. 2, we show the time evolution of the coupled modes as well as that of a single helicity mode. Here, we have defined the energy related to $\psi, \phi, V_{\|}$, and $\mathrm{p}$ of modes $(\mathrm{m}, \mathrm{n})$ as $E_{\psi}^{(m, n)}$ $=\int\left|\nabla \psi^{(m, n)}\right|^{2} d^{3} x, E_{\phi}^{(m, n)}=\int\left|\nabla \phi^{(m, n)}\right|^{2} d^{3} x, E_{V_{\|}}^{(m, n)}=\int V_{\|}^{(m, n)^{2}}$ $d^{3} x$ and $E_{p}^{(m, n)}=\left|\int p^{(m, n)} d^{3} x\right|$, respectively. We see a distinct difference in their behaviour as shown in the top panel of Fig. 2. The coupled NTMs show oscillations in the time evolution of their energies, while there are no such oscillations in the time evolution of a single $(3,1)$ NTM. The bottom panel of Fig. 2 shows that coupled NTMs generate higher perpendicular flows. Note also that the saturation level of the singly evolving $(3,1)$ NTM is lower than when it is coupled
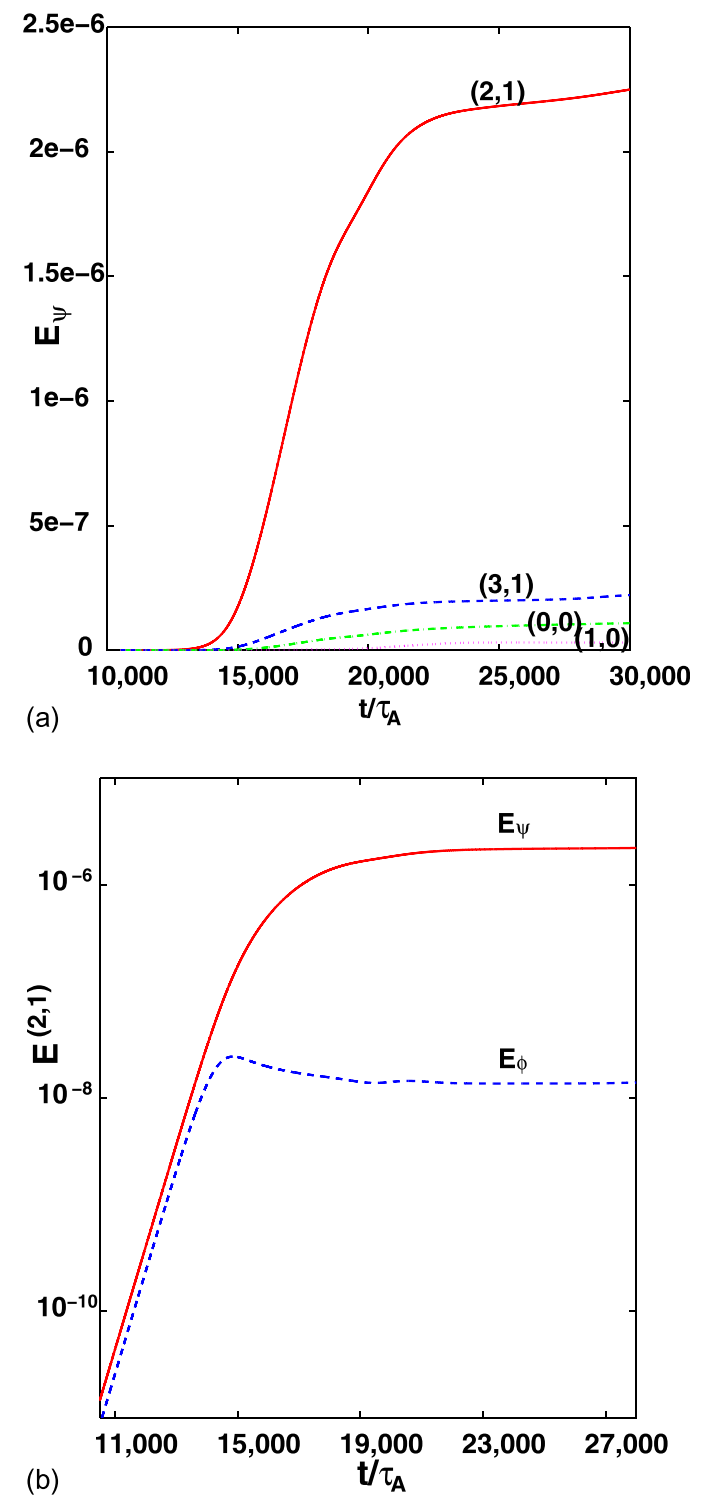

FIG. 3. Temporal evolution of magnetic energies for coupled tearing modes (TMs) (top panel) and comparison of magnetic and flow energies (bottom panel) for $\Delta^{\prime}>0, \mu_{e}=0$. to the $(2,1)$ mode. This is because the basic $(2,1)$ mode is more unstable than the $(3,1)$ mode for the equilibrium profile chosen in this case for the simulation.

To confirm that the oscillations have their origin in the neoclassical stress tensor term we have next carried out simulations with an equilibrium profile that is slightly unstable $\left(\Delta^{\prime}>0\right)$ to classical tearing modes in order to compare the behaviour of the modes when the neoclassical term is switched on or off. Fig. 3 shows that there are no oscillations in the energy evolution for coupled classical tearing modes, i.e., when $\mu_{e}=0$. In this case, the flow energy also remains small compared to the magnetic energy. The top panel of Fig. 5 shows that perpendicular flows are as usual restricted to their respective resonant surfaces.

Then when we switch on the neoclassical term (i.e., we take a finite $\mu_{e}$ ) for the same equilibrium we observe oscillations in the energy evolution as shown in Fig. 4. In this case, we also find higher values of the zonal magnetic fields (i.e.,
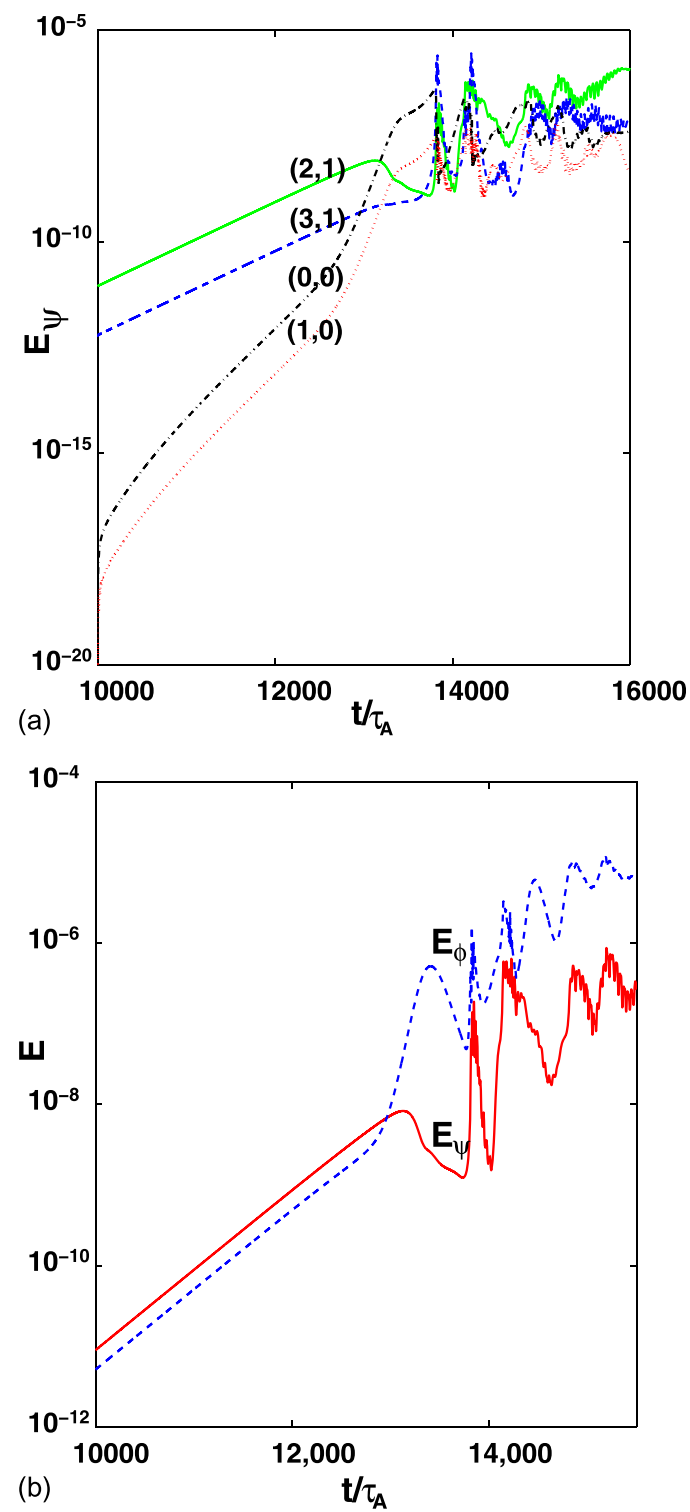

FIG. 4. Temporal evolution of magnetic energies with time for coupled TMs (top panel) and comparison of magnetic and flow energies (bottom panel) for $\Delta^{\prime}>0$, finite $\mu_{e}$. 


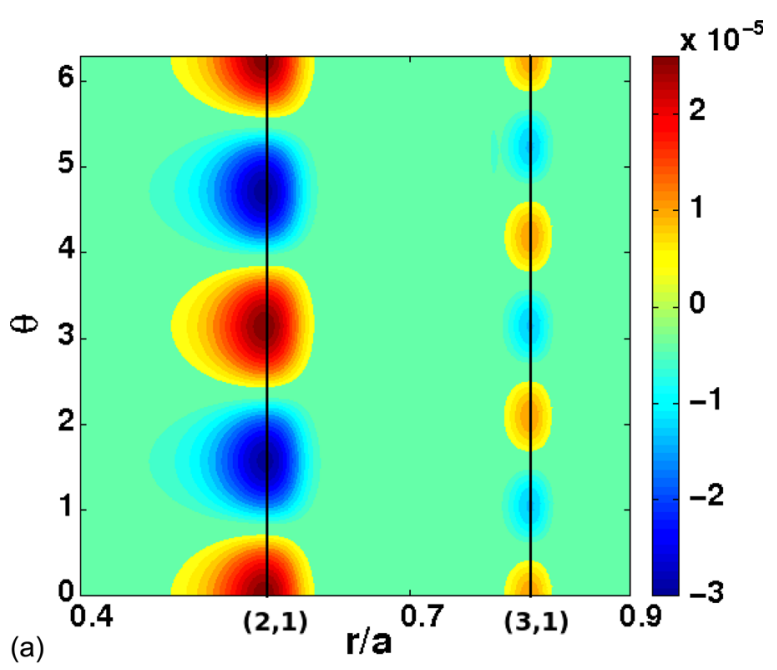

(a)

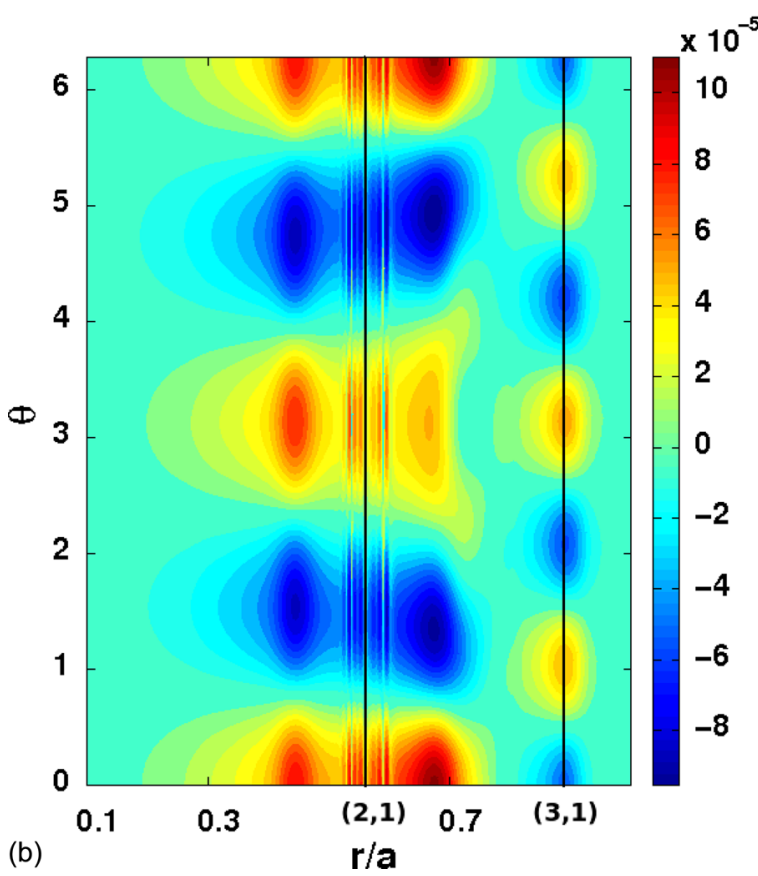

FIG. 5. $\phi$ contours of coupled tearing modes with $\Delta^{\prime}>0, \mu_{e}=0$ (top panel) and with $\Delta^{\prime}>0$, finite $\mu_{e}$ (bottom panel) at saturation.

$(0,0),(1,0)$ components) compared to the case for pure classical tearing modes. Fig. 4 shows the generation of higher perpendicular flows in the presence of a finite neoclassical viscous term. The bottom panel of Fig. 5 shows the equipotential contours of $\phi$. In contrast to the usual picture one has for pure classical tearing modes, we find that the flows are not restricted to their respective resonant surfaces but spread much beyond them. We also observe small scale flows inside the resonance layer. The top panel of Fig. 6 plot the magnetic flux surfaces using the Poincare plot which shows the $(2,1)$ and $(3,1)$ magnetic islands and the corresponding radial profiles of the electromagnetic potential $\Psi$ are shown in the bottom panel.

Next, we have tried to investigate the influence of parallel dynamics on the observed phenomena. As a simple measure, we have decoupled the $V_{\|}$dynamical equation from the Ohm's law and the vorticity equations (by dropping the terms proportional to $V_{\|}$in the latter two) and repeated the simulation runs for the coupled NTMs. Fig. 7 shows the
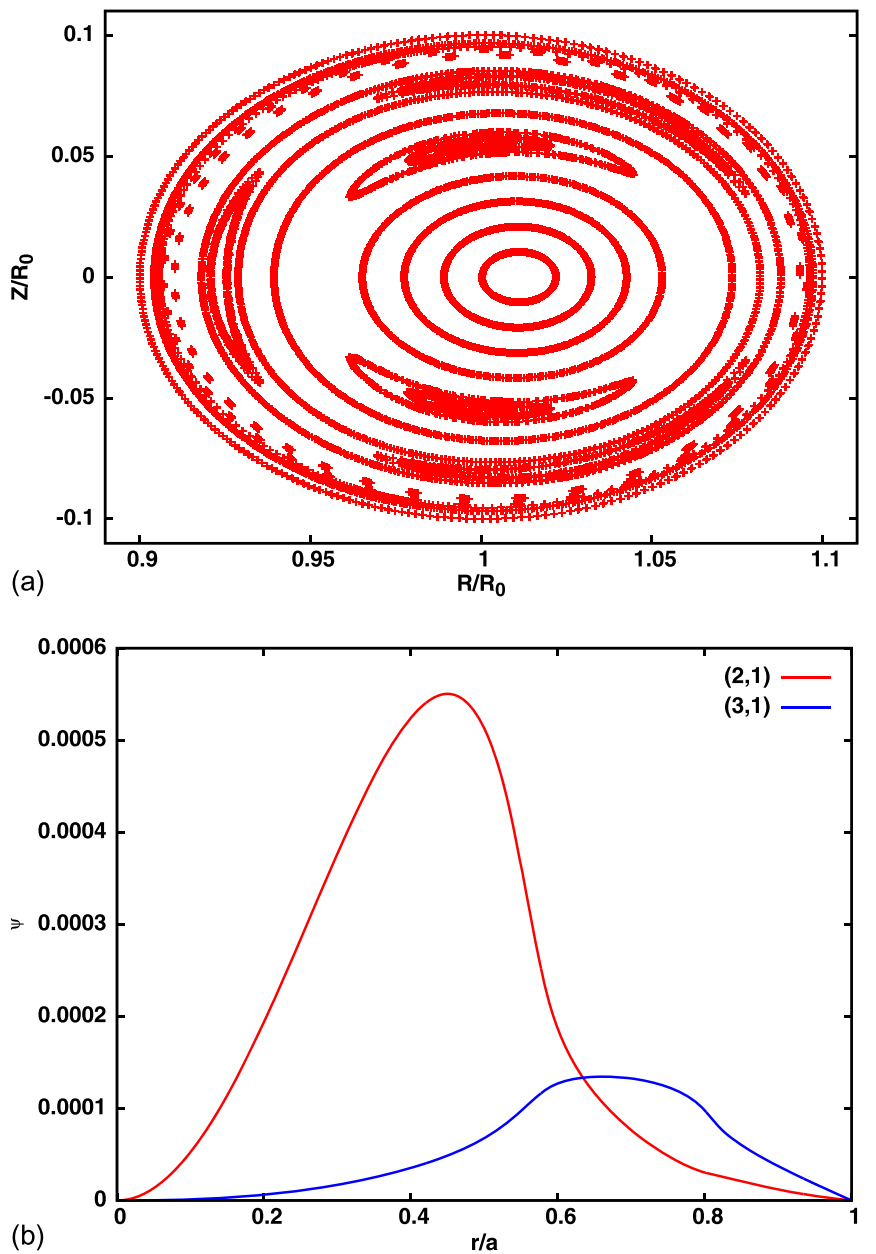

FIG. 6. Poincare plot showing the magnetic flux surfaces (top panel) and radial profiles of the electromagnetic potential $\Psi$ (bottom panel) for coupled tearing modes.

result of such a run and we notice that the oscillations in the energy have disappeared. This indicates that parallel dynamics plays an important role in the occurrence of oscillations.

To understand the nature of the oscillations a bit better, we have determined the characteristic frequencies of the oscillations from the power spectrum of the magnetic energy oscillations of the coupled NTMs. This is shown in the top panel of Fig. 8. Such spectra have been obtained for various values of $\beta$. Selecting the first harmonic peak of each of these spectra we have plotted the square of the oscillation frequencies for various values of $\beta$ as shown in the bottom panel of Fig. 8. A linear fit appears to best describe the dependence of the square of the frequency on $\beta$ indicating an acoustic origin of these oscillations. For comparison the curves with higher powers of $\beta$ are also shown in the same plot. Figs. 9 and 10 describe time evolutions of magnetic, flow, and pressure energies of the $(2,1),(3,1),(0,0)$, and $(1,0)$ modes. The enhanced values of the pressure components at the time of oscillations provide further evidence of the acoustic nature of the oscillations.

\section{DISCUSSION}

We have studied the mode coupling dynamics of two neoclassical tearing modes whose resonant surfaces are quite 

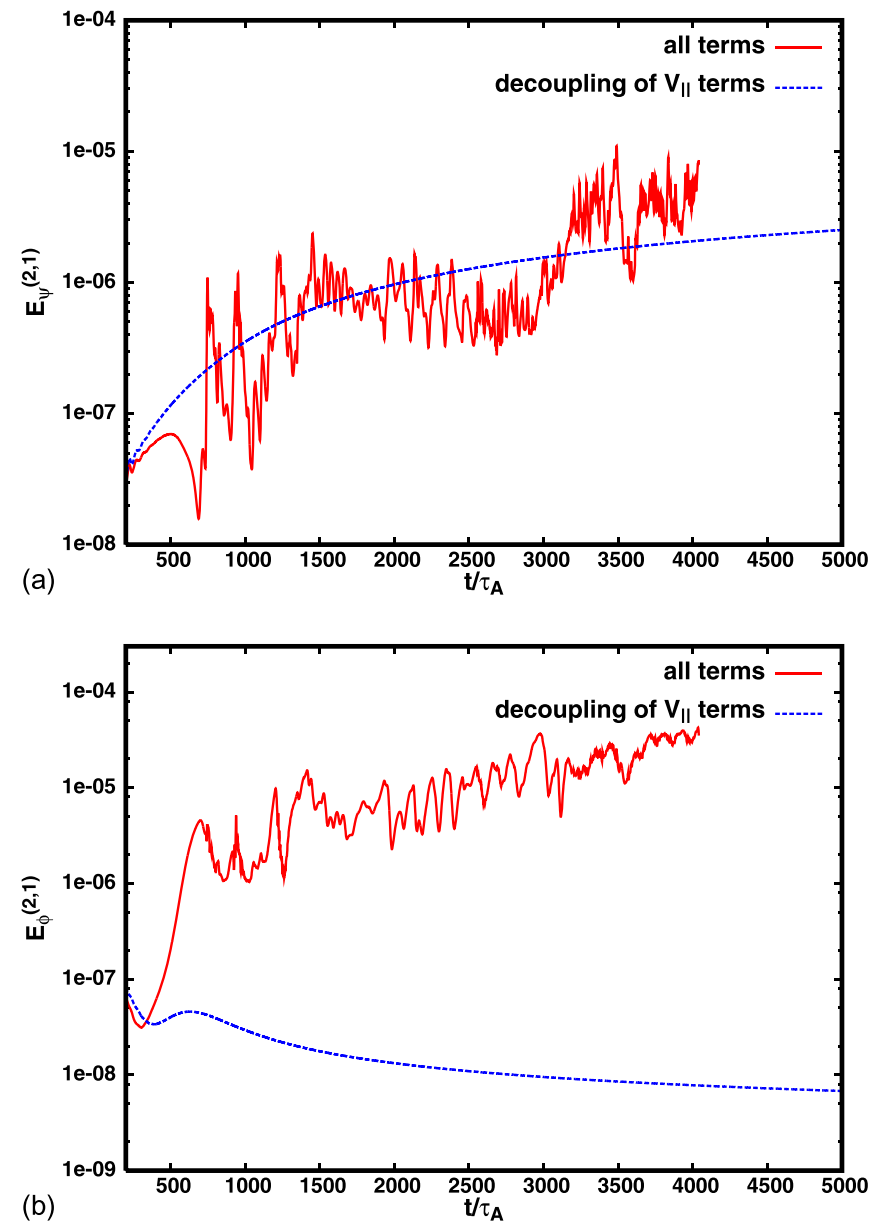

FIG. 7. Comparisons of the evolution of magnetic (top panel) and kinetic energies (bottom panel) for coupled NTMs by decoupling $V_{\|}$terms.

far apart. In contrast to past studies where the existence of a stochastic layer due to island overlap of closely spaced tearing layers enhances mode coupling effects we find a distinctly different and direct mechanism that has its origin in the presence of the neoclassical electron viscosity term in the Ohm's law. The presence of the neoclassical term leads to the excitation of oscillations in the mode energies, which is accompanied by an increase of flow energy and a substantial spreading of the flow beyond the resonant surfaces. The oscillations appear to be of an acoustic origin and are similar in nature to GAMs. The appearance of such oscillations are also accompanied by an increase in the energy content of the $(0,0)$ and $(1,0)$ equilibrium modes. The physical picture that emerges from a careful analysis of our numerical simulations is the following. A direct parametric interaction between the evolving $(2,1)$ and $(3,1)$ modes gives rise to perturbed neoclassical pressure contributions $\nabla p^{(0,0)}$ and $\nabla p^{(1,0)}$ in the Ohm's law. This in turn leads to the excitation of perturbed current components $J_{\|}^{(0,0)}$ and $J_{\|}^{(1,0)}$ and further leads to the generation of larger perpendicular flows through the contribution of the Maxwell stress tensor term $\left(B_{1} \cdot \nabla\right) J_{\|}$in the vorticity equation. The physical mechanism is somewhat analogous to what has been invoked for the interaction of magnetic islands with drift wave turbulence where the simultaneous excitation of modified GAMs along with large scale oscillatory vortices have been observed. ${ }^{14}$ The subsequent
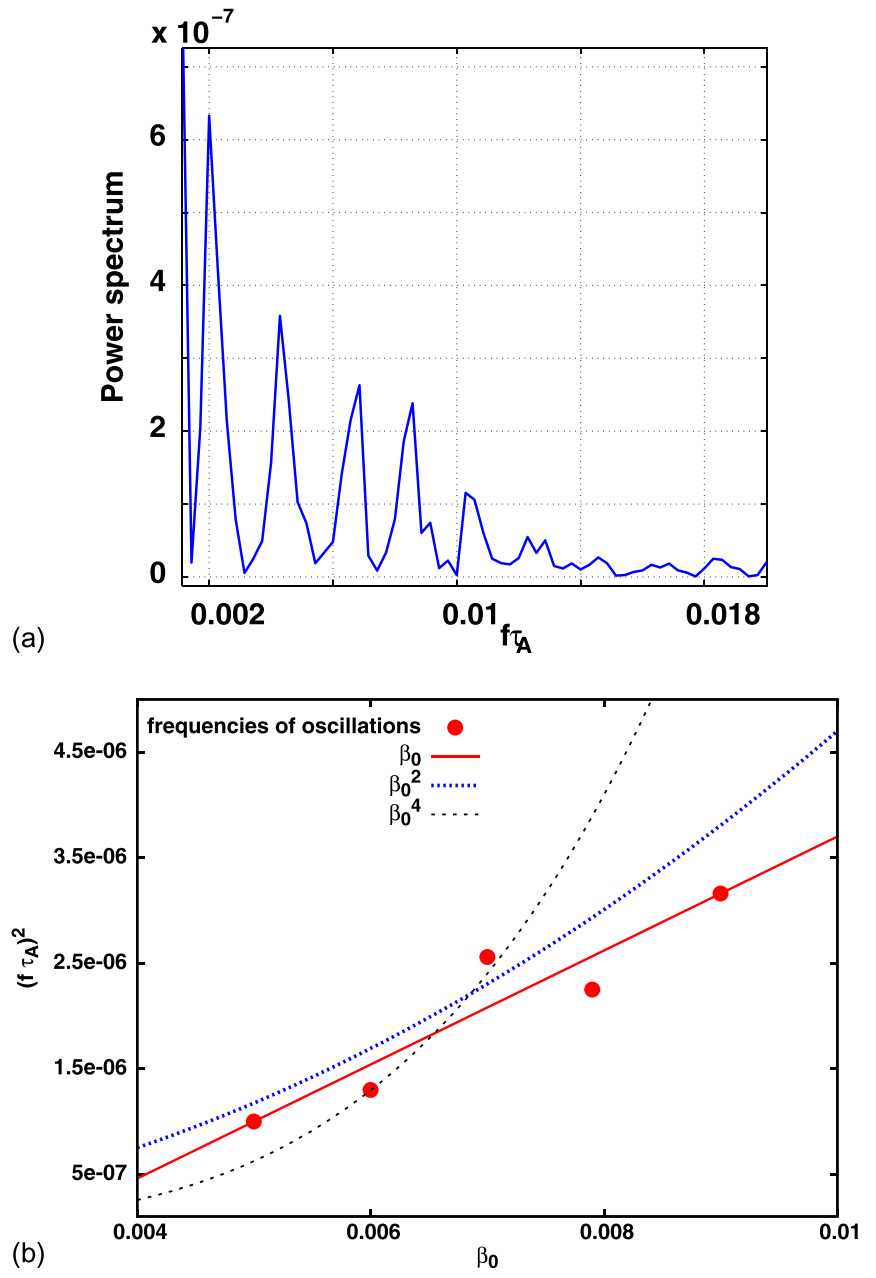

FIG. 8. Power spectrum of the oscillations in magnetic energies for $(2,1)$ coupled NTMs of Fig. 1 (top panel) and the filled circles in the plot on the bottom panel represent the square of frequencies of oscillations for different $\beta_{0}$ values. The lines are analytic curves that have a linear (red, solid), quadratic (blue, dotted) and a fourth power dependence on $\beta_{0}$ and have been drawn to show that the data are best represented by a linear fit.

compressional coupling between vorticity and parallel flow gives rise to perpendicular flows. In our case, as the numerical simulations show, it is the finite neoclassical electron viscosity term in the Ohm's law that permits flow of energy from the pressure gradient to the perpendicular flow through the involvement of the parallel current contribution. This mechanism is also consistent with past observations ${ }^{20}$ that zonal fields can only be generated with a term in Ohm's law that can break the frozen-in law. The electron neoclassical term in the Ohm's law that is proportional to $d p / d \psi$ serves that purpose and gives rise to the zonal fields at the resonant surfaces. We see clear evidence of this in the simulations from the enhanced energies in $\psi^{(0,0)}$ and $\psi^{(1,0)}$ field components as shown in Fig. 4.

To confirm the acoustic nature of the oscillations, we have obtained the frequency of oscillations from a power spectrum analysis of the time evolution of $E_{\psi}^{(2,1)}$ for coupled NTMs and found the dominant characteristic frequency of the oscillations to be close to that of a GAM. A typical GAM frequency can be approximately ${ }^{21,22}$ written as $\omega_{G A M}$ $\sim \frac{c_{s}}{R} \sim \frac{c_{s}}{V_{A} \tau_{A}}$, where $c_{s}=\sqrt{T_{e} / M}$ is the ion sound speed $\left(T_{e}\right.$ is 

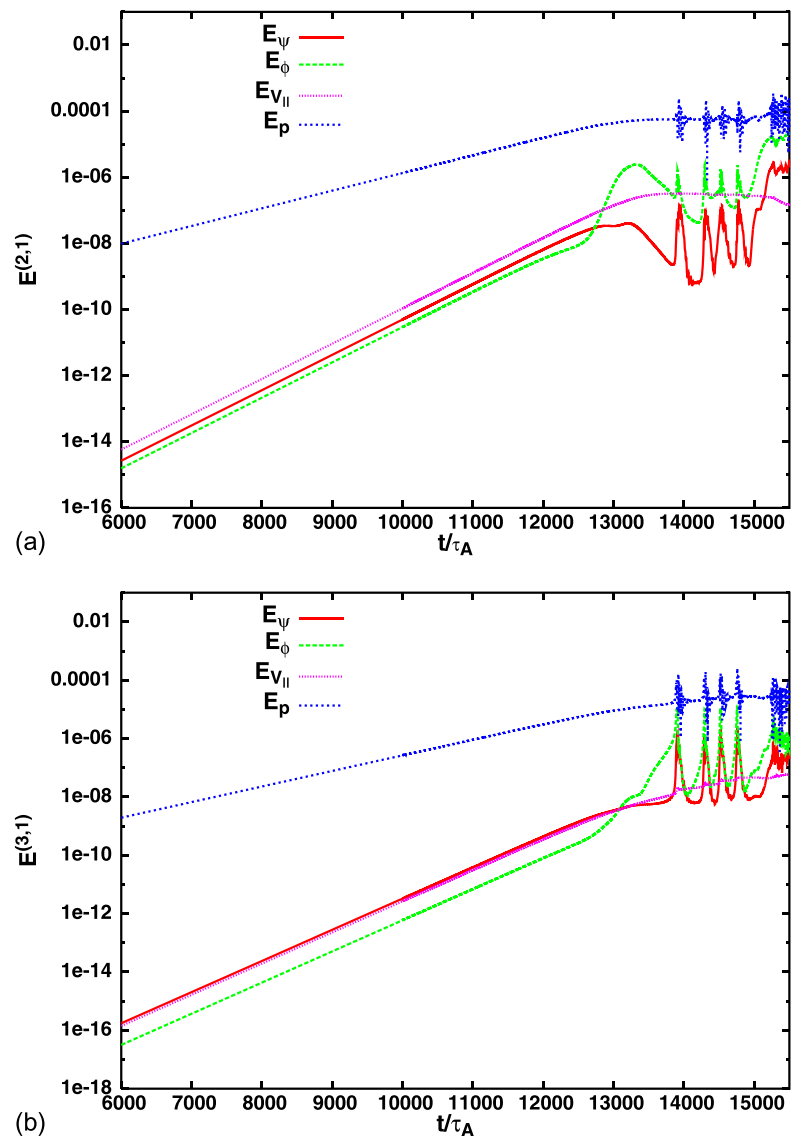

FIG. 9. Temporal evolution of $(2,1)$ modes (top panel) and $(3,1)$ modes of energies (bottom panel) of coupled TMs for $\Delta^{\prime}>0$, finite $\mu_{e}$.
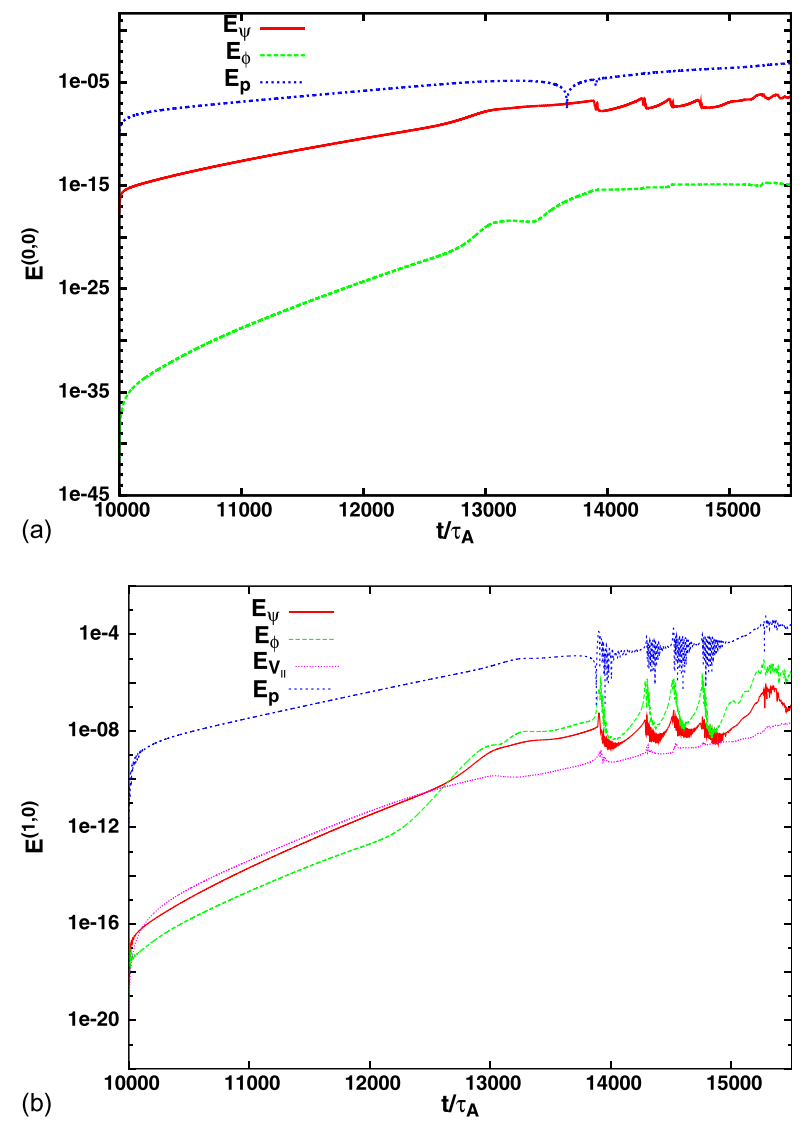

FIG. 10. Temporal evolution of $(0,0)$ modes (top panel) and $(1,0)$ modes of energies (bottom panel) of coupled TMs for $\Delta^{\prime}>0$, finite $\mu_{e}$. the electron temperature and $\mathrm{M}$ is the ion mass). Further, $V_{A}=\sqrt{B^{2} /(4 \pi \rho)}$ is the Alfvén velocity and $\tau_{A}$ is the Alfvén time. So the frequency $f_{G A M} \tau_{A} \sim \sqrt{\beta} / 2 \pi$. As discussed earlier the bottom panel of Fig. 8 showing the plot of the square of the characteristic frequencies of the oscillations for different $\beta$ values shows a linear trend indicating that these oscillations are likely to be of acoustic origin.

To conclude, our numerical simulation studies display a new and direct mode coupling mechanism between two NTMs of different helicities that can influence their nonlinear evolution. Apart from impacting their saturation levels the coupling induces GAM like oscillations that provide a more complex evolution signature for the NTMs. It would be interesting to identify such an effect experimentally particularly in situations where simultaneous existence of different helicity modes are observed.

\section{ACKNOWLEDGMENTS}

We would like to thank W. Horton, A. Smolyakov, and A. Thyagaraja for their valuable comments and D. Spong for his helpful suggestions on the numerics and for making available an upgraded integrator module for the code NEAR.

${ }^{1}$ T. C. Hender, J. C. Wesley, J. Bialek, A. Bondeson, A. H. Boozer, R. J. Buttery, A. Garofalo, T. P. Goodman, R. S. Granetz, Y. Gribov, O. Gruber, M. Gryaznevich, G. Giruzzi, S. Gnter, N. Hayashi, P. Helander, C. C. Hegna, D. F. Howell, D. A. Humphreys, G. T. A. Huysmans, A. W. Hyatt, A. Isayama, S. C. Jardin, Y. Kawano, A. Kellman, C. Kessel, H. R. Koslowski, R. J. La Haye, E. Lazzaro, Y. Q. Liu, V. Lukash, J. Manickam, S. Medvedev, V. Mertens, S. V. Mirnov, Y. Nakamura, G. Navratil, M. Okabayashi, T. Ozeki, R. Paccagnella, G. Pautasso, F. Porcelli, V. D. Pustovitov, V. Riccardo, M. Sato, O. Sauter, M. J. Schaffer, M. Shimada, P. Sonato, E. J. Strait, M. Sugihara, M. Takechi, A. D. Turnbull, E. Westerhof, D. G. Whyte, R. Yoshino, H. Zohm, and ITPA MHD, disruption and magnetic control topical group, Nucl. Fusion 47, S128 (2007).

${ }^{2}$ ITER Physics Expert Group on Disruptions, Plasma Control and MHD, ITER Physics Basis Editors, Nucl. Fusion 39, 2251 (1999).

${ }^{3}$ R. Carrera, R. D. Hazeltine, and M. Kotschenreuther, Phys. Fluids 29, 899 (1986).

${ }^{4}$ O. Sauter, R. J. La Haye, Z. Chang, D. A. Gates, Y. Kamada H. Zohm, A. Bondeson, D. Boucher, J. D. Callen, M. S. Chu, T. A. Gianakon, O. Gruber, R. W. Harvey, C. C. Hegna, L. L. Lao, D. A. Monticello, F. Perkins, A. Pletzer, A. H. Reiman, M. Rosenbluth, E. J. Strait, T. S. Taylor, A. D. Turnbull, F. Waelbroeck, J. C. Wesley, H. R. Wilson, and R. Yoshino, Phys. Plasmas 4, 1654 (1997).

${ }^{5}$ A. Gude, S. Gnter, S. Sesnic, and ASDEX Upgrade Team, Nucl. Fusion 39, 127 (1999).

${ }^{6}$ M. F. F. Nave, E. Lazzaro, R. Coelho, P. Belo, D. Borba, R. J. Buttery, S. Nowak, F. Serra, and EFDA-JET Contributors, Nucl. Fusion 43, 179 (2003).

${ }^{7}$ C. C. Hegna and J. D. Callen, Phys. Fluids B 4, 1855 (1992).

${ }^{8}$ Q. Yu and S. Gunter, Nucl. Fusion 39, 487 (1999).

${ }^{9}$ H. Lutjens and J.-F. Luciani, Phys. Plasmas 13, 112501 (2006).

${ }^{10}$ D. Raju, O. Sauter, and J. B. Lister, Plasma Phys. Controlled Fusion 45, 369 (2003)

${ }^{11}$ D. Chandra, A. Sen, P. Kaw, M. P. Bora, and S. Kruger, Nucl. Fusion 45, 524 (2005)

${ }^{12}$ M. Muraglia, O. Agullo, S. Benkadda, X. Garbet, P. Beyer, and A. Sen, Phys. Rev. Lett. 103, 145001 (2009).

${ }^{13}$ W. A. Hornsby, A. G. Peeters, A. P. Snodin, F. J. Casson, Y. Camenen, G. Szepesi, M. Siccinio, and E. Poli, Phys. Plasmas 17, 092301 (2010).

${ }^{14}$ W. A. Hornsby, A. G. Peeters, M. Siccinio, and E. Poli, Phys. Plasmas 19, 032308 (2012).

${ }^{15}$ S. E. Kruger, C. C. Hegna, and J. D. Callen, Phys. Plasmas 5, 4169 (1998).

${ }^{16}$ M. Baruzzo, B. Alper, T. Bolzonella, M. Brix, P. Buratti, C. D. Challis, F. Crisanti, E. de la Luna, P. C. de Vries, C. Giroud, N. C. Hawkes, 
D. F. Howell, F. Imbeaux, E. Joffrin, H. R. Koslowski, X. Litaudon, J. Mailloux, A. C. C. Sips, O. Tudisco, and JET-EFDA contributors, Plasma Phys. Controlled Fusion 52, 075001 (2010).

${ }^{17}$ T. A. Gianakon, C. C. Hegna, and J. D. Callen, Phys. Plasmas 3, 4637 (1996).

${ }^{18}$ See https://fusion.gat.com/THEORY/toq for TOQ, an equal-arc-length, inverse equilibrium solver for the Grad-Shafranov equation originally written by Bob Miller of General Atomics.
${ }^{19}$ D. Chandra, A. Sen, and P. K. Kaw, J. Plasma Fusion Res. 9, 457 (2010).

${ }^{20}$ F. Militello, M. Romanelli, J. W. Connor, and R. J. Hastie, Nucl. Fusion 51, 33006 (2011).

${ }^{21}$ N. Winsor, J. L. Johnson, and J. M. Dawson, Phys. Fluids 11, 2448 (1968).

${ }^{22}$ P. H. Diamond, S.-I. Itoh, K. Itoh, and T. S. Hahm, Plasma Phys. Controlled Fusion 47, R35 (2005). 